\title{
Phase- and Self-Injection-Locked Radar for Detecting Vital Signs with Efficient Elimination of DC Offsets and Null Points
}

\author{
Ping-Hsun Wu, Student Member, IEEE, Je-Kuan Jau, Chien-Jung Li, Member, IEEE, \\ Tzyy-Sheng Horng, Senior Member, IEEE, and Powen Hsu, Fellow, IEEE
}

\begin{abstract}
A phase- and self-injection-locked radar is presented for robust vital-sign detection. The dc offset from the clutter is eliminated by using a dual-tuning voltage-controlled oscillator without complex clutter cancellation techniques. Phase noise analysis shows that the proposed radar has the advantages of both phase-locked oscillators and self-injection-locked oscillators to achieve high power spectral signal-to-noise ratio. Path-diversity transmission is employed to eliminate null points and reduce average transmitted power. Experiments demonstrate successful detection of breathing and heartbeat from a distance of $4 \mathrm{~m}$ with $-22 \mathrm{dBm}(6 \mu \mathrm{W})$ average transmitted power.
\end{abstract}

Index Terms-DC offset, Doppler radar, null points, path-diversity, phase-locked oscillator, self-injection-locked oscillator, vitalsign detection.

\section{INTRODUCTION}

$\mathbf{T}$ HERE have been growing research activities on physiological sign detection techniques based on microwave Doppler radars for health-assistance devices, sleep apnea syndrome alarms, and life detectors for earthquake rescue [1]-[20]. The vibration of the chest wall due to cardiopulmonary activity modulates the phase of the signal reflected off the subject. Doppler radars extract the information of the respiration and heartbeat rates from the phase variation of the reflected signal. Early studies on the continuous-wave $(\mathrm{CW})$ radars for vital-sign

Manuscript received July 09, 2012; revised October 05, 2012; accepted October 16, 2012. Date of publication December 20,2012; date of current version January 17, 2013. This work was supported by the National Science Council, Taiwan, under Contract NSC 100-2221-E-002-221-MY2. This paper is an expanded paper from the IEEE MTT-S International Microwave Symposium, Montreal, QC, Canada, June 17-22, 2012.

P.-H. Wu is with the Graduate Institute of Communication Engineering, National Taiwan University, Taipei 10617, Taiwan, and the Information and Communications Research Laboratories, Industrial Technology Research Institute, Hsinchu 31040, Taiwan (e-mail: pinghsunwu@itri.org.tw).

J.-K. Jau is with the Information and Communications Research Laboratories, Industrial Technology Research Institute, Hsinchu 31040, Taiwan (e-mail: eastpeak@gmail.com).

C.-J. Li is with the Department of Electronic Engineering, National Taipei University of Technology, Taipei 106, Taiwan (e-mail: chnjung@ntut.edu.tw).

T.-S. Horng is with the Department of Electrical Engineering, National Sun Yat-Sen University, Kaohsiung 80424, Taiwan (e-mail: jason@ee.nsysu.edu. tw).

P. Hsu is with the Department of Electrical Engineering and the Graduate Institute of Communication Engineering, National Taiwan University, Taipei 10617, Taiwan (e-mail: phsu@cc.ee.ntu.edu.tw).

Color versions of one or more of the figures in this paper are available online at http://ieeexplore.ieee.org.

Digital Object Identifier 10.1109/TMTT.2012.2228222 detection have used phase-locked oscillators due to better phase noise [2], [3]. The development of range correlation theory makes free-running oscillators applicable only for short range vital-sign monitoring because the signal-to-noise ratio (SNR) degrades rapidly with increasing detection distance [4]. Moreover, the flicker noises from the mixers in the conventional Doppler radar receiver further degrade the detection sensitivity [5].

The dc offset due to the clutter and circuit imperfections is a critical issue for conventional $\mathrm{CW}$ radars since it saturates and desensitizes the receiver. Various clutter cancellation and dc offset calibration techniques have been reported with complex RF front-end and digital signal processing (DSP) hardware [7], [8]. In [9], a heterodyne receiver has been presented to reduce the dc offset from local oscillator leakage. However, the coupling from transmitter to receiver results in interferences at the same IF frequency could not be removed. Moreover, the residual phase noise from additional local oscillator degrades the detection sensitivity. A Doppler radar using a self-injection-locked (SIL) oscillator has been proposed to achieve a high SNR gain in the vital-signal bandwidth [10]. However, it suffers from uncontrolled frequency drift which causes electromagnetic interference (EMI) issues in practical applications.

The null detection problem encountered at every quarter wavelength distance from the radar to the subject is another critical issue for reliable vital-sign detection. The quadrature demodulation architecture is the most prevailing solution while the extra flicker noise contributed by the mixers degrades the detection accuracy [4]. Double-sideband and two-tone transmission systems require multiple signal sources with frequency tuning algorithm to find the optimal detection point when the subject changes its position [9], [11]. Arctangent demodulation method [12] combines the in-phase and quadrature baseband signals into a single channel to eliminate the null points. However, it requires complicated calibrations on the dc offsets for accurate demodulation. Complex signal reconstruction approach [13] is less sensitive to the dc offset while the clutter reflection inevitably degrades the detection sensitivity in the mixer-based receiver. Millimeter wave radars have been developed with shorter wavelength to avoid the null detection problem [14], [15]. However, the design is still challenging with respect to robustness, power consumption, and cost. Radar with passive harmonic tag has been proposed to isolate the target from clutter for improving the SNR [16]. However, the tag attached on the body discomforts the user. The detection 
distance is short due to the passive harmonic power conversion. The time-domain detection techniques such as ultra-wide band (UWB) radars are free from the null detection problem with complex time discriminators [17]. Nevertheless, they fail to detect the vital-signs when the strong clutter has identical round-trip time to the Doppler signal, unless complicated signal processing algorithms for clutter reduction are adopted [21], [22]. Other works including antenna-diversity radars and frequency-diversity radars have been reported to eliminate the null points at the expense of duplicating multiple sets of the RF front-end circuits [18]. Single channel receivers using the dynamic phase variation technique have been proposed to find the optimal detection point with complicated algorithm [18], [19]. However, the strong reflection from clutter could also block the radar receiver from finding the optimal point.

In the authors' preliminary work [23], a phase- and self-injection-locked (PSIL) oscillator has been developed for vital-sign detection. The innovative method obtains the vital-sign signal by directly sampling the voltage-controlled oscillator (VCO) tuning voltage controlled by the phase-locked loop (PLL) without any demodulation circuits. However, the dc offset problems caused by the clutter and circuit imperfections were not addressed in regard to the reliable detection. In this paper, we present a simple and hardware efficient method to eliminate the dc offset using a dual-tuning VCO and avoiding the complex clutter cancellation circuits. Analysis based on the classic injection locking equation shows that the dc offset can be eliminated without any frequency drift and sensitivity degradation. In addition, the phase noise analysis based on the linear frequency-domain model shows that the proposed radar takes advantages of phase-locked oscillators and SIL oscillators with low phase noise and high power spectral SNR gain, respectively. Furthermore, the null points are eliminated by the proposed path-diversity transmitter that switches between two orthogonal SIL paths without occupying extra bandwidth. The average transmitted power is reduced by adjusting the duty cycle. A prototype circuit is designed, fabricated and tested to check the accuracy of the design equations. Experimental results show that successful detections of the respiration and heartbeat rates can be obtained up to 4 meters distance with $-22 \mathrm{dBm}$ average transmitted power, when the radar wave is emitted toward the front side of the chest wall.

\section{OPERATION PRINCIPLE OF THE PSIL RADAR}

Fig. 1 displays the system block diagram of the proposed Doppler radar based on the PSIL oscillator with the path-diversity switch. The dual-tuning VCO has the fine tuning voltage $\left(V_{T}\right)$ controlled by the PLL to extract the Doppler phase modulated signal and the coarse tuning voltage $\left(V_{A D J}\right)$ is set by the control circuit to eliminate the dc offset. The received Doppler signal is injected into the VCO through the circulator to form an SIL loop. Then, the SIL loop is phase locked by the PLL to stabilize the output frequency and reduce the in-band phase noise. The Doppler injection signal results in an output phase perturbation of the $\mathrm{VCO}$ which is detected by the phase frequency detector (PFD). The charge pump (CP) circuit and the loop filter

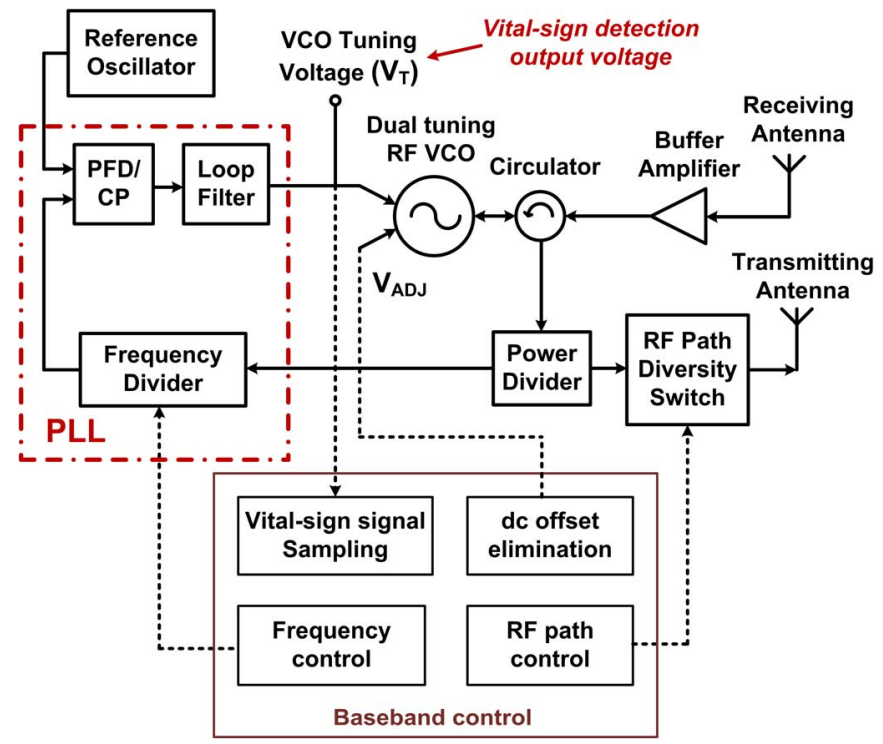

Fig. 1. System block diagram of the PSIL Doppler radar with path-diversity switch.

transform the output of the PFD into a voltage for tuning the intrinsic oscillation frequency of the VCO. As a result, the VCO fine tuning voltage $V_{T}$ controlled by the PLL is the vital-sign output voltage that reflects the phase variation of the Doppler signal. The path-diversity switch transmitter periodically alters the transmission path to eliminate the null points and reduce the average transmitted power.

The steady-state analysis provides comprehensive derivation for the operation of the PSIL oscillator since the vital-sign signal is slowly varying. The injection locking phenomena has been reported in the literatures [24]-[26]. When the VCO is injection locked by a sinusoidal signal $I_{i n j}$ with a frequency $\omega_{i n j}$ deviating from the intrinsic oscillation signal $I_{o s c}$ with a tank resonant frequency $\omega_{o s c}$, the injection signal forces the resonant tank to generate an additional phase shift to meet the Barkhausen oscillation criterion. The instantaneous output signal $I_{\text {out }}$ can be obtained by the vector sum of $I_{i n j}$ and $I_{o s c}$ as depicted in Fig. 2(a), where $\omega_{\text {out }}$ represents the instantaneous output signal frequency, $\theta$ is the phase difference between $I_{i n j}$ and $I_{o s c}$, and $\psi$ is the phase difference between $I_{o u t}$ and $I_{o s c}$. It has been shown [25] that

$$
\sin \theta=\frac{\sqrt{I_{o s c}^{2}+I_{i n j}^{2}+2 I_{o s c} I_{i n j} \cos \theta}}{I_{i n j}} \sin \psi \cong \frac{\omega_{o s c}-\omega_{i n j}}{\omega_{L R}}
$$

where the one-sided locking range can be determined as

$$
\omega_{L R}=\frac{\omega_{o s c}}{2 Q} \cdot \frac{I_{i n j}}{I_{o s c}} \cdot \frac{1}{\sqrt{1-\left(\frac{I_{i n j}^{2}}{I_{o s c}^{2}}\right)}} \cong \frac{\omega_{s y n}}{2 Q} \sqrt{\frac{P_{i n j}}{P_{o s c}}}
$$

under weak injection approximation. In (2), $P_{i n j}$ is the power injected into the $\mathrm{VCO}, P_{o s c}$ is the $\mathrm{VCO}$ free-running oscillation power, and $Q$ is the quality factor of the VCO resonant tank. Since the VCO is phase locked by the PLL, the instantaneous output frequency is the synthesized frequency $\omega_{s y n}=N \omega_{\text {ref }}$, 


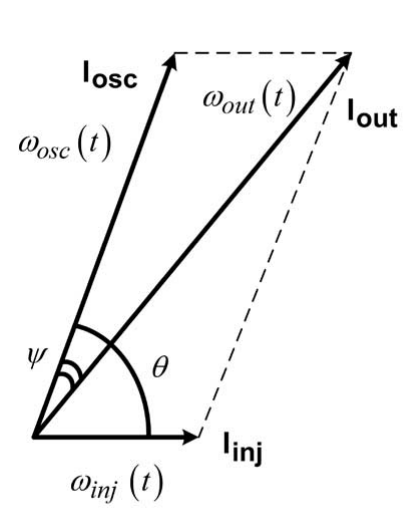

(a)

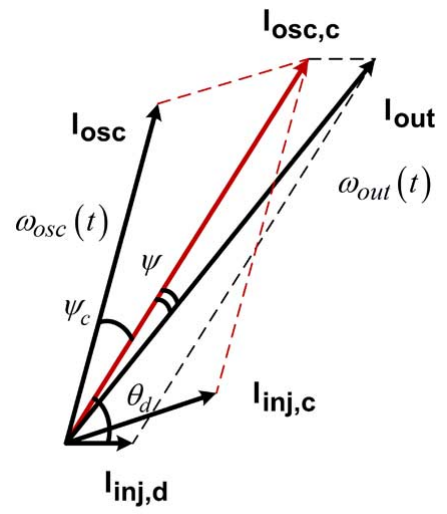

(b)
Fig. 2. Vector diagram of the instantaneous current signals in the SIL oscillator. (a) with injection signal. (b) with clutter and injection signals.

where $\omega_{\text {ref }}$ is the frequency of the reference oscillator and $N$ is the divider modulus.

When there is a stationary object within the detection range, the reflected signal injected into the VCO establishes an SIL loop. The signals in the SIL oscillator can be illustrated by Fig. 2(a), where $I_{i n j}$ represents the injection signal reflected from the stationary object. The free-space round-trip delay and circuit delay result in a phase difference $\theta$ between $I_{i n j}$ and $I_{o s c}$. When the SIL oscillator is phase locked, it follows from (1) that

$$
\omega_{\text {out }}=\omega_{\text {inj }}=\omega_{\text {osc }}-\omega_{L R} \sin \theta=\omega_{\text {syn }}
$$

in which $\omega_{L R}$ denotes the one-sided locking range of the injection signal reflected from the stationary object and $\omega_{o s c}$ is determined from the tuning voltages of the resonant tank, which can be represented by

$$
\omega_{o s c}=\omega_{o s c, 0}+K_{V} V_{T}+K_{V, A D J} V_{A D J}=\omega_{s y n}+K_{V} \Delta V_{T}
$$

where $\omega_{o s c, 0}$ is the VCO tank resonant frequency with both tuning voltages at zero and $K_{V}$ and $K_{V . A D J}$ are the corresponding VCO gains with respect to the fine tuning voltage $V_{T}$ and the coarse tuning voltage $V_{A D J}$, respectively. In the following analysis, $V_{T}$ and $V_{A D J}$ are set to preset values for a specified synthesized frequency by the dc offset elimination circuit described in the next section. The reflected Doppler signal injected into the VCO produces a variation of the tuning voltage $\Delta V_{T}$ controlled by the PLL. Since $\omega_{\text {out }}$ and $\omega_{i n j}$ remain constant when the SIL oscillator is phase locked in a steady state, the variation of the $\mathrm{VCO}$ fine tuning voltage due to the stationary object can be derived from (3) and (4) as

$$
\Delta V_{T}=\frac{\omega_{L R} \sin \theta}{K_{V}} .
$$

This equation of the PSIL radar indicates that the reflected signal from the stationary object results in a variation on the VCO tuning voltage controlled by the PLL with respect to the locking range and the phase difference between $I_{i n j}$ and $I_{o s c}$. In the proposed architecture, $V_{T}$ is the output voltage containing the Doppler information. The output voltage variation is periodic with respect to the distance of the stationary object away

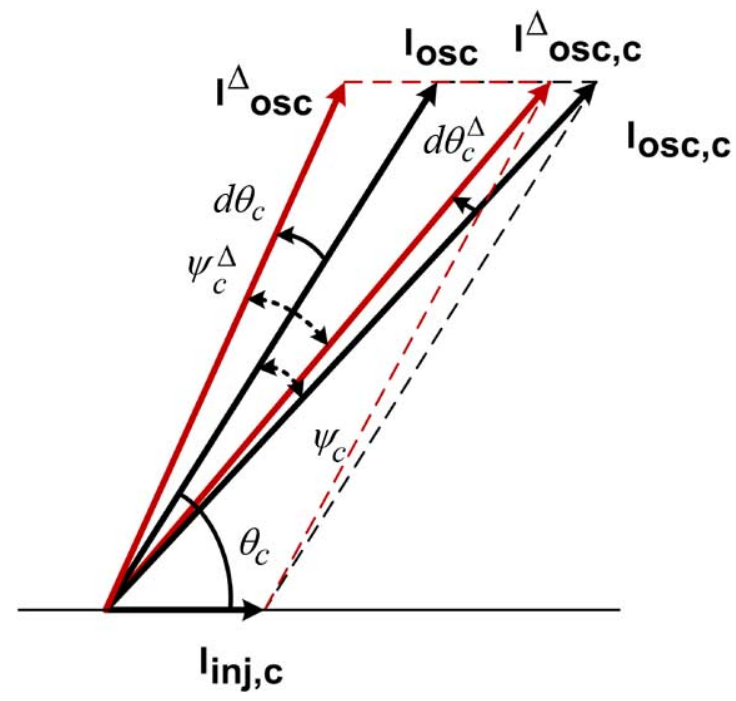

Fig. 3. Vector diagram of the current signals in the SIL oscillator with a perturbation of the VCO tuning voltage under clutter injection.

from radar since $\theta$ accounts for the round-trip phase delay. The period is a half free-space wavelength. Furthermore, the VCO gain $K_{V}$ can be used to increase the detection sensitivity without high-gain signal amplification.

\section{Clutter Effect and CAncellation Technique}

\section{A. Detection Sensitivity Under Clutter Injection}

The clutters, including the coupling between the antennas, circuit reflections, and stationary reflecting and scattering objects within the detection range, degrade the detection sensitivity in the conventional Doppler radars. The proposed PSIL radar can maintain the detection sensitivity with strong clutter. Fig. 2(b) displays the vector diagram of the PSIL oscillator with both the strong clutter signal $I_{i n j, c}$ and the weak Doppler signal $I_{i n j, d}$. The signal $I_{o s c, c}$ is the sum of $I_{o s c}$ and $I_{i n j, c}$, representing the effective VCO oscillation signal under clutter injection. The phase difference $\theta_{d}$ between $I_{i n j, d}$ and $I_{o s c, c}$ stands for the round-trip delay of the signal reflected from the target.

The clutter effect on the detection sensitivity can be analyzed using Fig. 3. The effective VCO oscillation signal $I_{o s c, c}$ under clutter injection causes a change of dc level in the VCO tuning voltage, which can be equivalently modeled as a variation in the VCO gain. Let $I_{o s c}^{\Delta}$ denote the VCO's intrinsic oscillation signals with a small tuning voltage perturbation $d V_{T}$. The signal $I_{o s c, c}^{\Delta}$ is the sum of $I_{o s c}^{\Delta}$ and $I_{i n j, c}$, representing the variation from $I_{o s c, c}$ under clutter injection. The angle $\psi_{c}$ is the phase difference between $I_{o s c, c}$ and $I_{o s c}$, whereas $\psi_{c}^{\Delta}$ is the phase difference between $I_{o s c, c}^{\Delta}$ and $I_{o s c}^{\Delta}$. With the relationship $I_{o s c} \psi_{c}=I_{i n j, c} \sin \theta_{c}$ as shown in [25], if there is a small perturbation $d \theta_{c}$ due to a small VCO tuning voltage variation $d V_{T}$, the variation of the angle $d \theta_{c}^{\Delta}$ can be obtained as

$$
\begin{aligned}
d \theta_{c}^{\Delta} & =d \theta_{c}-\left(\psi_{c}^{\Delta}-\psi_{c}\right)=d \theta_{c}-\frac{I_{i n j, c}}{I_{o s c}}\left[\sin \left(\theta_{c}+d \theta_{c}\right)-\sin \left(\theta_{c}\right)\right] \\
& \cong\left[1-\left(\frac{I_{i n j, c}}{I_{o s c}} \cos \theta_{c}\right)\right] d \theta_{c} .
\end{aligned}
$$


The variation of the VCO tuning voltage $d V_{T}$ causes a different output signal when clutter injection exists. Since the VCO gain stands for the change of the resonant frequency with respect to the tuning voltage, the clutter effect can be regarded as a variation to the VCO gain. One can obtain the effective VCO gain $K_{V, c}$ under clutter injection as

$$
K_{V, c}=K_{V}\left(\frac{\frac{d \theta_{c}^{\Delta}}{d V_{T}}}{\frac{d \theta_{c}}{d V_{T}}}\right) \cong K_{V}\left(1-\frac{I_{i n j, c}}{I_{o s c}} \cos \theta_{c}\right)
$$

Now consider the proposed radar for detecting the cardiopulmonary activity with strong clutter. In this case, the amplitude of the Doppler echo signal $I_{i n j, d}$ is smaller than that of the clutter $I_{i n j, c}$, as shown in Fig. 2(b). The steady state equation of the PSIL radar can be derived as

$$
\omega_{o u t}=\omega_{i n j}=\omega_{o s c, c}+K_{V, c} \Delta V_{T, d}-\omega_{L R, d} \sin \theta_{d}
$$

where $\omega_{o s c, c}$ is the intrinsic oscillation frequency of the PSIL oscillator with clutter and $\omega_{L R, d}$ is the one-sided locking range of the Doppler injection signal from the cardiopulmonary activity. Similarly, $\omega_{\text {out }}$ remains constant when the radar is phase locked in a steady state. The variation of the VCO tuning voltage $\Delta V_{T, d}$ due to the Doppler signal can be derived from (8) as

$$
\Delta V_{T, d}=\frac{\omega_{L R, d} \sin \theta_{d}}{K_{V, c}} .
$$

The physiological movement due to the respiration and heartbeat can be regarded as a periodic variation with average distance $d_{0}$. The round-trip time delay is denoted as $\tau_{d}$ and the corresponding phase delay is $\phi_{d}$. According to the Doppler theory, when there is a small chest wall movement from the cardiopulmonary activity, the phase of the reflected signal can be found as

$$
\theta_{d}(t)=\frac{2 \omega_{s y n}}{c} x_{\text {body }}(t)+\phi_{d}
$$

where $x_{b o d y}(t)$ is the instantaneous position shift from $d_{0}$ and $c$ is the speed of light. The VCO tuning voltage variation $\Delta V_{T, d}$ with respect to $x_{\text {body }}(t)$, which is regarded as the detection sensitivity of the PSIL radar, can be derived as

$$
\frac{-\partial \Delta V_{T, d}}{\partial x_{b o d y}}=\frac{-\omega_{s y n}^{2} \cos \phi_{d}}{K_{V, c} Q c} \sqrt{\frac{P_{i n j}}{P_{o s c}}} .
$$

The above analysis based on the classic injection locking equations shows the detection sensitivity is retained in the PSIL radar with the clutter effect. Eq. (11) can be used to calculate the circuit parameters of the PSIL radar for a specified detection sensitivity requirement. The detection sensitivity is proportional to the square root of the ratio of the injection power to the intrinsic oscillation power. The PSIL radar retains the property of the SIL radar [10] that the sensing distance can grow four times longer by doubling the operating frequency. It is also inversely proportional to the quality factor and the VCO gain. The detection sensitivity can be further tuned by adjusting the VCO gain or adding a variable gain amplifier in the receiver chain.

\section{B. DC Offset Elimination Technique Using Dual-Tuning VCO}

The dc offset in the mixer-based conventional Doppler radars requires complex cancellation and calibration circuits in both the RF front-end and baseband. This paper presents a simple and hardware efficient technique to eliminate the dc offset using the dual-tuning VCO. For nominal operation of the PSIL radar, the fine tuning voltage $\left(V_{T}\right)$ controlled by the PLL is used to extract the vital-sign signal with a small $K_{V}$ to obtain high detection sensitivity. However, the PSIL radar with high detection sensitivity produces considerably large dc offset when the clutter reflection signal is injected into the VCO. The radar may fail to detect small cardiopulmonary activity under strong clutter injection because the dc offset may saturate the sampling circuit. It is intuitive that the dc offset can be mitigated by tuning the PLL synthesized frequency to shift the output voltage back to the desired dc level. This frequency tuning technique can be applied against the dc offset problem in the PSIL radar. However, the frequency tuning technique has an undetermined bandwidth since the operating frequency is forced to vary in different clutter environments. Moreover, the voltage tuning step cannot be well controlled since it is also affected by the clutter injection, as shown in (5). The frequency change causes a variation of $\theta$, resulting in the shift of the dc offset.

In this paper, the dc offset can be easily eliminated by adjusting the coarse VCO tuning voltage $V_{A D J}$, which is controlled by the dc offset elimination circuit. The clutter injection forces the VCO resonant tank to change the intrinsic oscillation frequency and results in the dc offset given by (5). In the proposed dual-tuning $\mathrm{VCO}$, the intrinsic oscillation frequency is determined by both $V_{T}$ and $V_{A D J J}$, as shown in (4). When there is a dc level variation $\Delta V_{T, \text { Clutter }}$ in $V_{T}$, the coarse VCO tuning voltage $V_{A D J}$ can be adjusted to eliminate the dc level variation by the following equation:

$$
K_{V} \Delta V_{T, \text { Clutter }}+K_{V, A D J} \Delta V_{A D J}=0
$$

where the adjustment voltage of the coarse tuning port $\Delta V_{A D J}$ can be given as

$$
\Delta V_{A D J}=-\frac{K_{V}}{K_{V, A D J}} \Delta V_{T, \text { Clutter }} .
$$

Typically the VCO gain value of $K_{V, A D J}$ is larger than that of $K_{V}$ to simultaneously obtain larger frequency channel selection range and high detection sensitivity. This ensures that the dc offset can be always eliminated even if $\Delta V_{T, \text { Clutter }}$ saturates the sampling circuit. It is noted that the detection sensitivity is not affected by $K_{V, A D J}$ since after dc offset elimination, $V_{A D J}$ is a fixed constant value under the specified operating condition. Compared to the conventional dc offset cancellation architectures, the proposed technique is simple and hardware efficient without any bulky RF attenuators, phase shifters, and complex DSP clutter cancellation algorithms.

\section{Phase NoISE ANAlysis}

The Laplace domain analysis is a popular and classic method to examine the noise performance of a PLL. A linear frequencydomain model is constructed to evaluate the SNR performance 


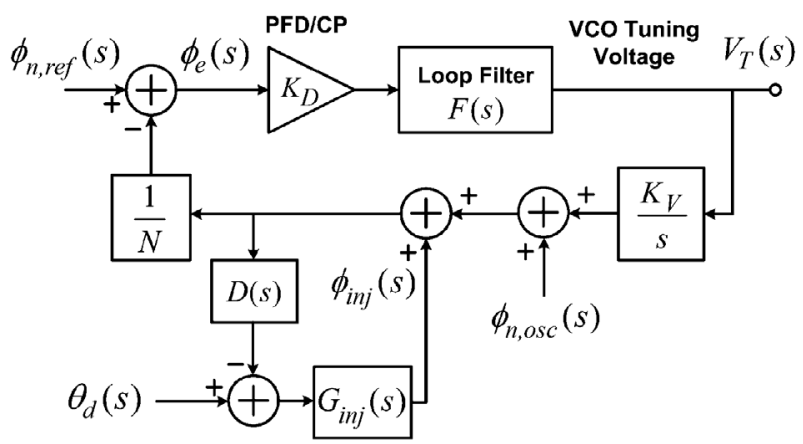

Fig. 4. Linear frequency domain model for phase noise analysis.

of the proposed radar, as shown in Fig. 4. The Doppler phase modulated signal is denoted as $\theta_{d}(s)$, which is the Laplace transform of $\theta_{d}(t)$. The phase noise from the reference crystal oscillator is represented by $\phi_{n, r e f}(s)$. The intrinsic VCO phase noise is denoted as $\phi_{n, o s c}(s)$. The noises in the active circuits including the PFD, CP, frequency divider, and loop filter are ignored since they have little contributions to the low-frequency offset phase noise. The gain of the PFD and CP circuits is denoted as $K_{D} . F(s)$ stands for the transfer function of the loop filter.

The PLL inherently serves as the demodulator for the Doppler phase shift. The vital-sign signal which is the variation of the VCO tuning voltage controlled by the PLL can be derived as $V_{T}(s)=K_{D} F(s) \phi_{e}(s)$. The excess phase $\phi_{e}(s)$ at the PFD output is given by

$$
\phi_{e}(s)=\phi_{n, r e f}(s)-\frac{1}{N}\left[\phi_{i n j}(s)+\phi_{n, o s c}(s)+\frac{K_{V}}{s} V_{T}(s)\right]
$$

where $\phi_{i n j}(s)$ is the equivalent phase injection signal in an SIL loop. It has been shown that the SIL mechanism can be represented as a delta-sigma model [10]. The transfer function of the SIL loop can be found as

$$
T(s)=\frac{1}{1-G_{i n j}(s) D(s)}
$$

in which $G_{i n j}(s)=\omega_{L R} \cos \phi_{d} / s$ represents the transfer function of the injection process and $D(s)=1-\exp \left(-s \tau_{d}\right)$ represents the transfer function of the round-trip propagation path with delay $\tau_{d}$. The overall transfer function including the Doppler signal and the phase noises can be expressed as

$$
\begin{aligned}
V_{T}(s)=H_{n, r e f}(s) \phi_{n, r e f}(s)+H_{n, o s c}(s) & \phi_{n, o s c}(s) \\
& +H_{D}(s) \theta_{d}(s)
\end{aligned}
$$

under the assumption that the Doppler signal and the phase noise sources are uncorrelated. In (16), the transfer function of the VCO tuning voltage controlled by the PLL with respect to the reference oscillator phase noise can be derived as

$$
H_{n, r e f}(s)=\frac{s K_{D} F(s)}{s+\frac{K_{D} F(s) K_{V} T(s)}{N}} .
$$

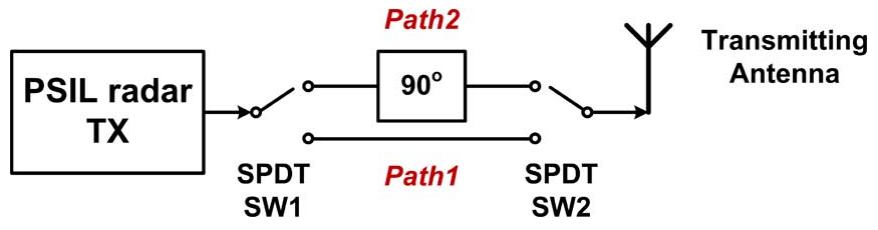

Fig. 5. Mechanism of the path-diversity transmission.

The transfer function of the VCO tuning voltage controlled by the PLL with respect to the intrinsic VCO phase noise is

$$
H_{n, o s c}(s)=\frac{-s K_{D} F(s) \frac{T(s)}{N}}{s+\frac{K_{D} F(s) K_{V} T(s)}{N}}
$$

The variation of the VCO tuning voltage controlled by the PLL due to the Doppler phase modulation can be found as

$$
H_{D}(s)=\frac{-\omega_{L R} \cos \phi_{d} K_{D} F(s) \frac{T(s)}{N}}{s+\frac{K_{D} F(s) K_{V} T(s)}{N}} .
$$

Equations (17) and (18) show that the PSIL radar suppresses in-band phase noises from both reference oscillator and VCO. The advantage of high power spectral SNR gain in the free-running SIL oscillator with respect to the intrinsic VCO phase noise is retained. Moreover, the in-band phase noise of a phase-locked oscillator is dominated by reference crystal oscillator and divider modulus of the PLL, which can be obtained as $N \phi_{n, r e f}(s)$. Therefore, phase noise of a PSIL oscillator is superior to that of a free-running SIL oscillator. The PSIL radar has a power spectral SNR gain against the conventional radars with phase-locked oscillators. From (17) and (19), this power spectral SNR gain is obtained as

$$
G_{S N R}(s)=\frac{\left|H_{D}(s)\right|^{2}}{\left|H_{n, \text { ref }}(s)\right|^{2}} \cdot N^{2}=\left|\frac{\omega_{L R} \cos \phi_{d}}{s}\right|^{2}
$$

when the Laplace variable $s$ is small. This equation is the same as (21) in [10], therefore the proposed PSIL radar has both the advantages of low phase noise from the phase-locked and high power spectral SNR gain from the SIL oscillators. As a result, the PSIL radar can serve for long-range detections with less transmitted power.

\section{Path Diversity TRANSMission}

The CW radars suffer from the null detection problem that exists at every multiple of a quarter free-space wavelength distance. The PSIL radar also has the null detection problem since it has a zero power spectral SNR gain and fails to detect the physiological movement when $\cos \phi_{d}=0$ in (11) and (20). In this paper, we propose a single channel receiver topology to eliminate the null points using the path-diversity transmission, as shown in Fig. 5. The two signal paths have a difference of a $90^{\circ}$ phase shift. From (11), the detection sensitivity of the first path is proportional to $\cos \phi_{d}$ while that of the second path with additional $90^{\circ}$ phase shift is proportional to $\sin \phi_{d}$. The two SIL paths are periodically switched to ensure that when one is in the null point, the other will be in the optimal point. 


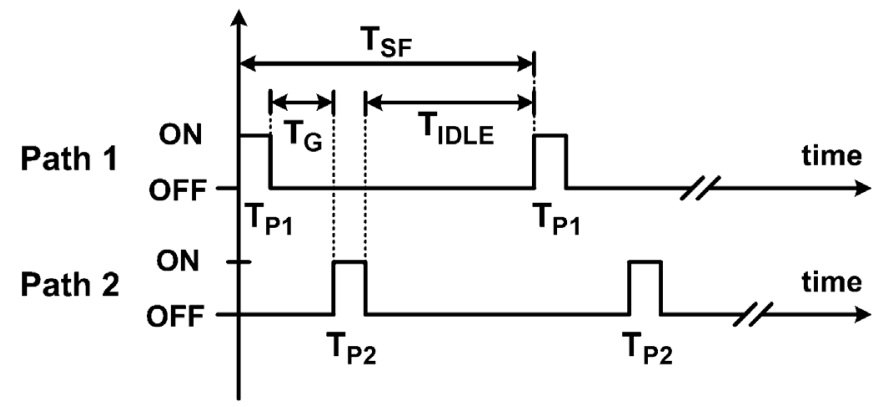

Fig. 6. Control timing diagram of the path-diversity switch.

Another advantage of the path-diversity transmission is to reduce the average transmitted power by repetitively enabling the signal transmission within short pulse durations for the concern of human electromagnetic energy exposure. The control timing diagram is shown in Fig. 6. The period of a sensing period is denoted as $T_{S F}$. There are two pulses with duration $T_{P 1}$ and $T_{P 2}$ in a sensing period to alternately switch on the two SIL paths. The path control is synchronized with the receiver sampling circuit. A guard time interval $T_{G}$ mitigates the noise from the transition between the SIL loops. The vital-sign output voltage is sampled only when stable SIL loops are established. The average transmitted power can be reduced while the detection sensitivity is not affected since the vital-sign signal has a slow time-varying waveform.

The steady-state vital-sign output voltages of each SIL path including the clutter effects can be obtained by (5) and (9). The dc offsets can be then removed using the proposed dual-tuning VCO technique. The settling time of the transition from an idle state to an SIL steady state is determined by both the injection locking stabilization time and the PLL settling time for correcting the phase error introduced by the injection signals. The time constant of the injection locking stabilization process is the inverse of the locking range $1 / \omega_{L R}$ under weak injection condition. The other dominant factor of the settling time is the PLL settling time, which is related to the damping factor $\zeta$ and the natural frequency $\omega_{n}$ of a typical second-order PLL. The PLL settling time can be determined by designing the loop filter and the supply current of the $\mathrm{CP}$ circuit. It is noted that the divider modulus of PLL is not altered and the output frequency is stable. The required settling time is less than $0.2 \mathrm{~ms}$ at a natural frequency of several kilohertz. For accurate detection of the cardiopulmonary activity, $T_{S F}$ must be designed by the Nyquist criterion. The detection pulses, $T_{P 1}$ and $T_{P 2}$, must be designed to obtain steady-state SIL operations.

\section{EXPERIMENTAL RESULTS}

Experiments have been conducted in the laboratory to verify the feasibility of the proposed PSIL radar for vital-sign detection. Fig. 7 displays the hybrid prototype circuit for the experiments. The hybrid dual-tuning Colpitts VCO is implemented with an Avago ATF-55143 enhancement-mode pseudo-HEMT. The free-running frequency and the oscillation power of the $\mathrm{VCO}$ is $2.41 \mathrm{GHz}$ and $-5 \mathrm{dBm}$, respectively. The loaded quality factor of the $\mathrm{VCO}$ is 22 , which can be determined by measuring the locking range of the VCO. The VCO gains $K_{V}$ and $K_{V, A D J}$

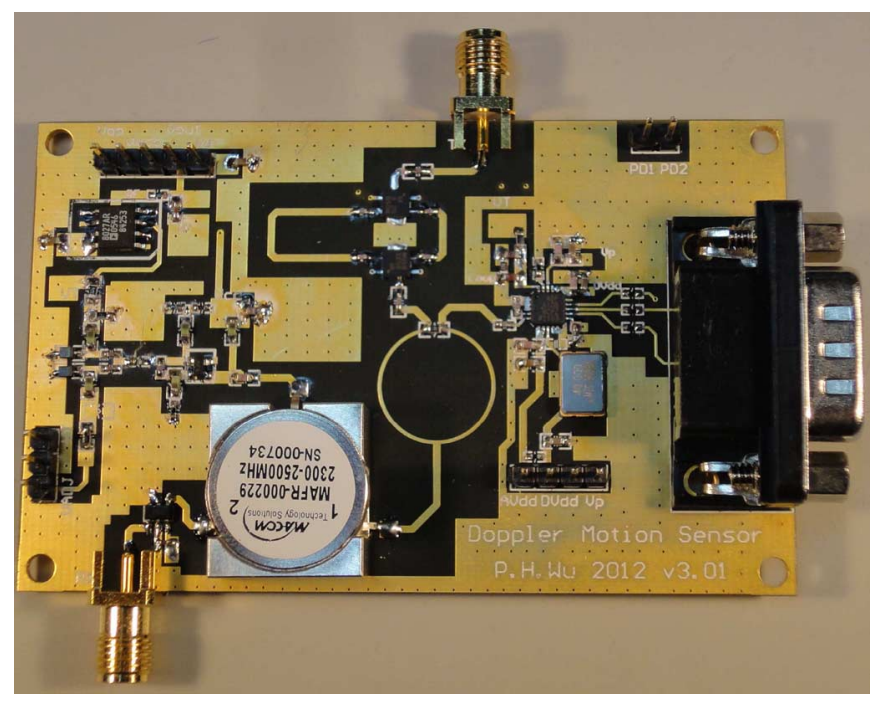

Fig. 7. Photograph of the hybrid prototype circuit.

are set to 2 and $40 \mathrm{MHz} / \mathrm{V}$, respectively. The power fed to the transmitting antenna in a selected SIL path is $-11 \mathrm{dBm}$ with the loss of the power divider, the circulator, and the path-diversity switch circuit. The MA-COM surface mount circulator has $25 \mathrm{~dB}$ isolation, $0.2 \mathrm{~dB}$ insertion loss and $25 \mathrm{~dB}$ return loss. Two RFMD RF3025DS non-reflective SPDT T/R switches are employed in the path-diversity switch circuit with terminated off-state to eliminate internal circuit reflections. The insertion losses of the two orthogonal signal paths are slightly different. The first path has a $3.1 \mathrm{~dB}$ loss while the other path with additional quarter-wavelength delay has a $3.4 \mathrm{~dB}$ loss owing to the lossy FR4 substrate. The phase difference is $88.5^{\circ}$ due to the fabrication process variation.

Both the transmitting and receiving antennas are commercially available with $12 \mathrm{dBi}$ gain and $120^{\circ}$ beamwidth. The buffer amplifier at the receiver has a $13 \mathrm{~dB}$ gain. The reflected Doppler signal is injected into the VCO through the circulator. The VCO is phase locked with a commercial Analog Devices ADF4108 PLL integer-N frequency synthesizer IC. A quartz crystal oscillator generates the reference frequency at $40 \mathrm{MHz}$. The steady-state output voltage is set to a half of the CP supply voltage for largest voltage-swing range using the dc offset elimination technique.

\section{A. Verification of the Design Equation}

Fig. 8 shows the experimental setup to verify (5). A copper plate with dimensions of $12.5 \mathrm{~cm} \times 10 \mathrm{~cm}$ is placed in front of the antennas. Eq. (5) indicates that the output voltage is a periodic function with a half of the free-space wavelength period.

The copper plate is initially placed at a $60 \mathrm{~cm}$ distance from the antennas, satisfying the far-field condition. It is gradually moved away from the antennas at $0.5 \mathrm{~cm}$ step to a $76 \mathrm{~cm}$ distance. The measured output voltages are shown in Fig. 9. The solid line is the theoretical prediction with $2 \mathrm{MHz} / \mathrm{V} \mathrm{VCO}$ gain. The dashed line is the theoretical prediction with the same circuit parameters and an additional $90^{\circ}$ phase delay. The amplitude is slightly different from the solid line due to the additional $0.3 \mathrm{~dB}$ loss of the path-diversity switch circuit. The measured 


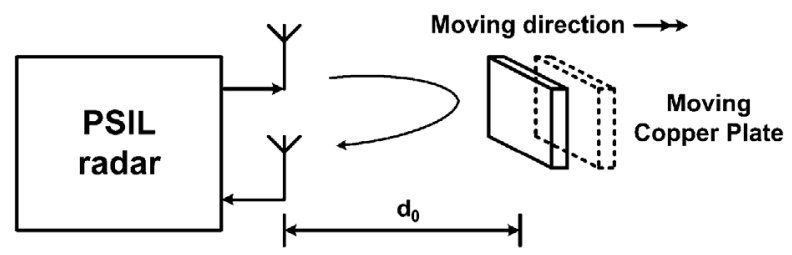

Fig. 8. Experimental setup to measure the output voltage of the PSIL radar for detecting a copper plate moving from 60 to $76 \mathrm{~cm}$ with a step of $0.5 \mathrm{~cm}$.

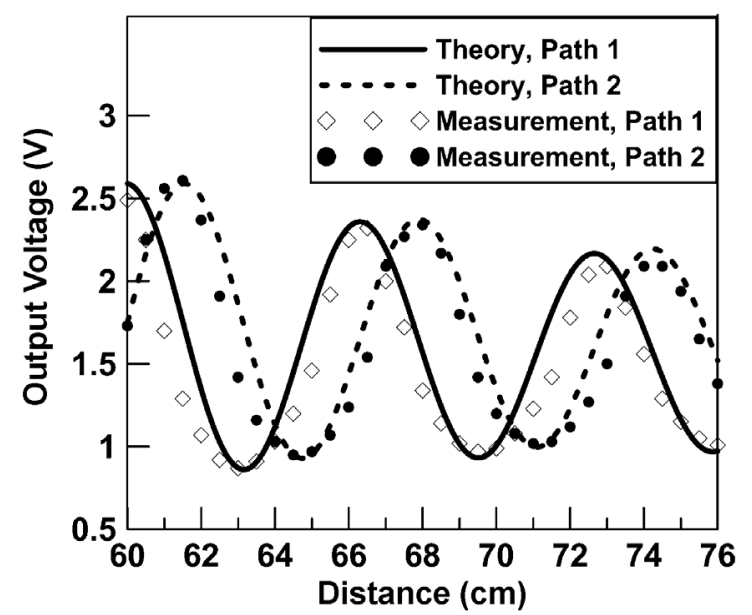

Fig. 9. Measured output voltages of the PSIL radar for detecting a copper plate moving from 60 to $76 \mathrm{~cm}$ with a step of $0.5 \mathrm{~cm}$.

output voltage with respect to the position of the copper plate is periodic with a $6.2 \mathrm{~cm}$ period, which is accurately a half of the free-space wavelength. Good agreement is observed between theoretical and experimental results.

\section{B. Detection Sensitivity Under Clutter Injection}

As discussed in Section III-A, the detection sensitivity of the PSIL radar is not obviously affected under strong clutter interference. Two experiments are performed to consider different clutters from electromagnetic wave reflection and circuit coupling. Fig. 10(a) shows the experimental setup to measure the detection sensitivity under clutter reflection within the detection area. A copper plate with dimensions of $50 \mathrm{~cm} \times 50 \mathrm{~cm}$ is placed beside the moving copper plate as a stationary object for producing clutter. The measured reflected signal power from the clutter is larger than that of a moving copper plate by $12 \mathrm{~dB}$. Both the copper plates are initially placed at a $120 \mathrm{~cm}$ distance from the antennas. In this experiment, the clutter copper plate remains at a fixed position and the moving copper plate is moved away from the antennas at a $0.5 \mathrm{~cm}$ step. Fig. 11 compares the results between measured output voltages with and without the clutter. The dc offset due to clutter is eliminated by the proposed dual-tuning VCO. In Fig. 11, the measured output voltage with respect to the position of the moving copper plate is still periodic with a period of $6.2 \mathrm{~cm}$ and the measured peak-to-peak output voltages with and without clutter are both $0.29 \mathrm{~V}$. Thus

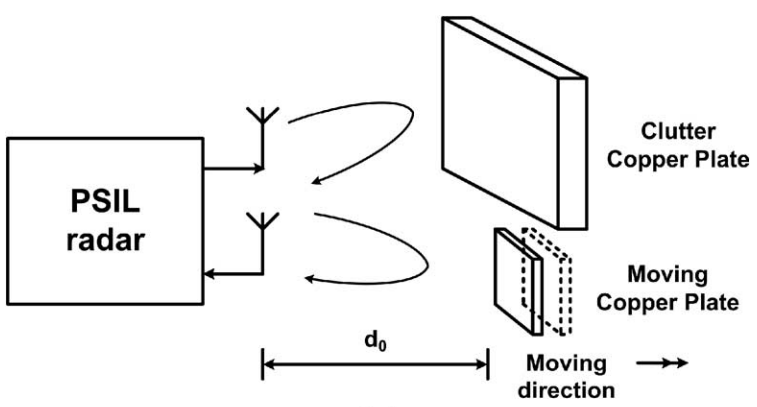

(a)

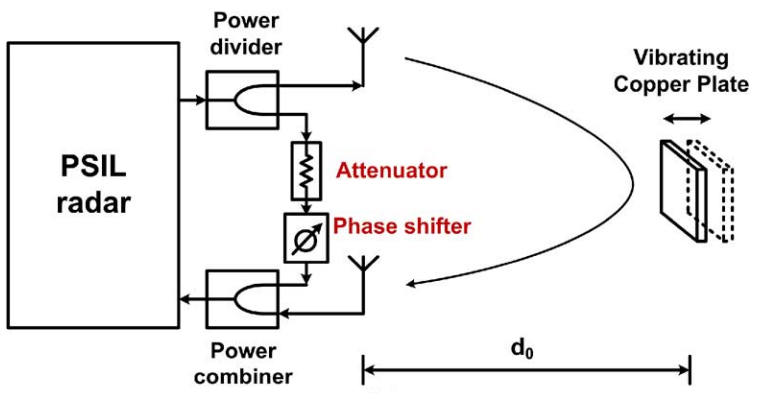

(b)

Fig. 10. Experimental setups to measure the output voltage of the PSIL radar. (a) with nearby clutter (b) with circuit coupling.

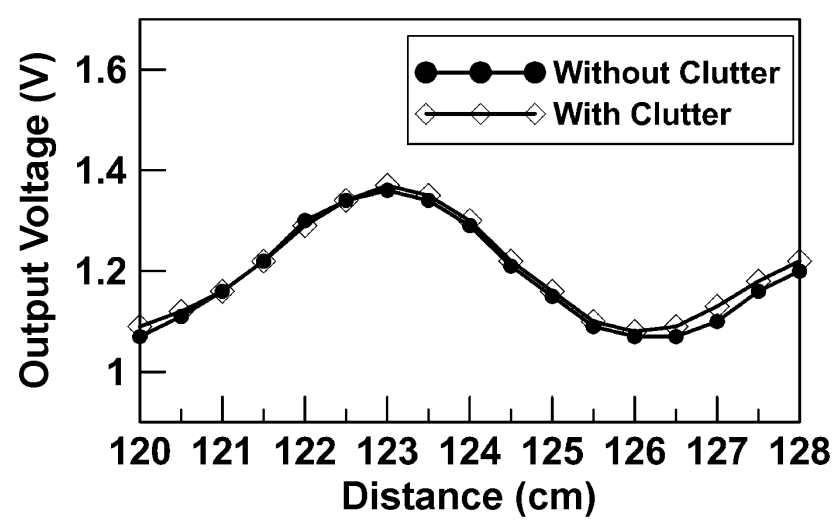

Fig. 11. Measured output voltages of the PSIL radar with and without nearby clutter.

the output voltage is obviously not affected by the clutter, as predicted by (7) and (9).

Fig. 10(b) shows the experimental setup to imitate generation of the clutter from internal circuit coupling. For this purpose, a coupling circuit that is composed of an attenuator and an adjustable phase shifter is used in the coupling path. The transmitted power is divided equally to feed the transmitting antenna and the coupling circuit. The copper plate with dimensions of $12.5 \mathrm{~cm} \times 10 \mathrm{~cm}$ is attached on a mechanical actuator to generate a stable Doppler phase-modulated signal. The vibration frequency is $3.2 \mathrm{~Hz}$ and the maximum displacement is $2 \mathrm{~mm}$. The Doppler and clutter signals are combined and then injected into the VCO. The attenuation levels used in the experiment are 20,30 and $40 \mathrm{~dB}$. The phase shifter is adjusted with a step of a $30^{\circ}$ interval. The measured voltages are normalized with the case without clutter. 


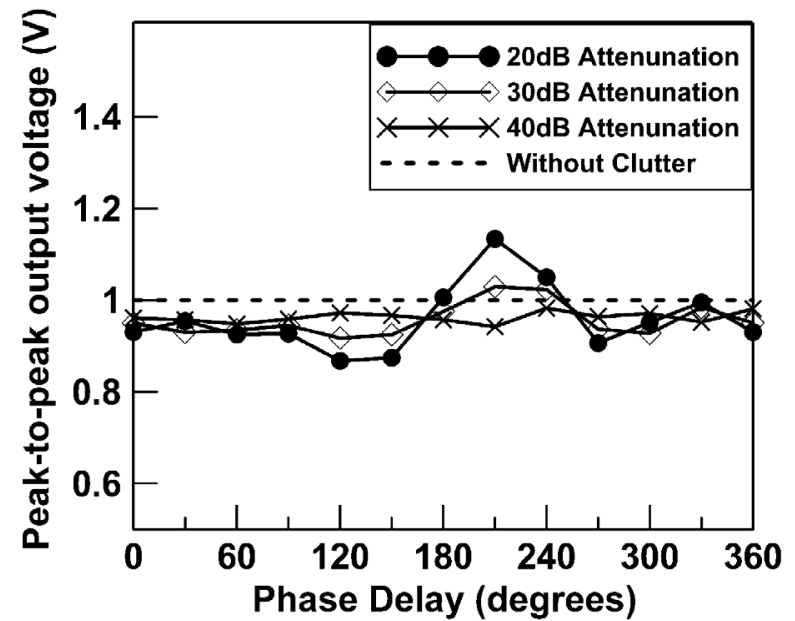

Fig. 12. Measured peak-to-peak output voltages of the PSIL radar with clutter from circuit coupling.

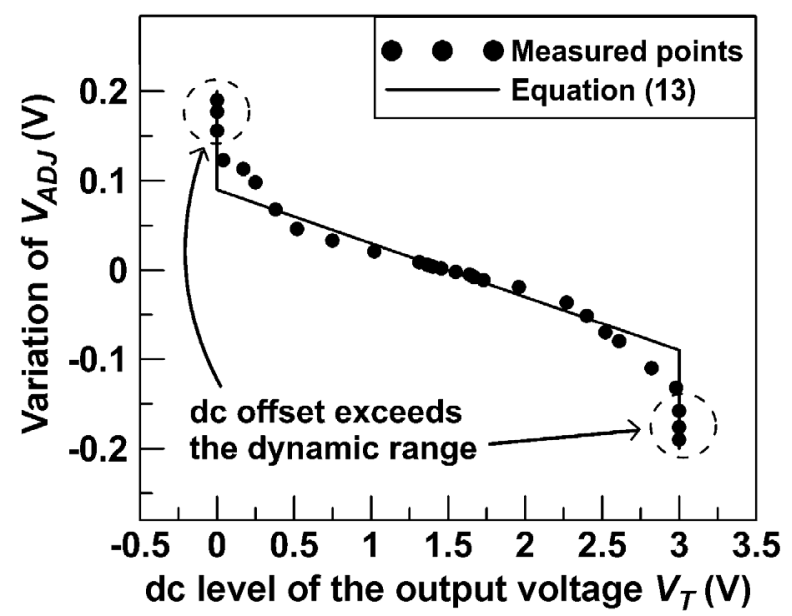

Fig. 13. Measured variation of the coarse tuning voltage required to eliminate the dc offset of the PSIL radar.

The measured peak-to-peak output voltages at $3.2 \mathrm{~Hz}$ are plotted in Fig. 12, which shows that the detection sensitivity remains regardless of the clutter from circuit coupling.

\section{Elimination of dc Offset From Clutter}

As discussed in Section III-B, the dc offset from clutter injection can be eliminated using the dual-tuning VCO. An experiment is performed to consider the different clutter-induced dc offset and the required $\Delta V_{A D J}$ for elimination. The coupling path in Fig. 10(b) is used to produce clutter with different dc offset values. Then, the dc offset is measured and eliminated by tuning $V_{A D J}$. In the experiment, the CP supply voltage is $3 \mathrm{~V}$ and the de level is set to $1.5 \mathrm{~V}$. Fig. 13 plots measured $\Delta V_{A D J}$ to eliminate the dc offset due to various clutter injections. The deviation of the measured points from theoretical prediction is due to the nonlinearity of the varactor. For the clutter injection which saturates the output voltage, the PSIL radar can also eliminate the dc offset by increasing $\Delta V_{A D J}$ more. This ensures that the dc offset can also be eliminated even if it exceeds the dynamic range of the sampling circuit.

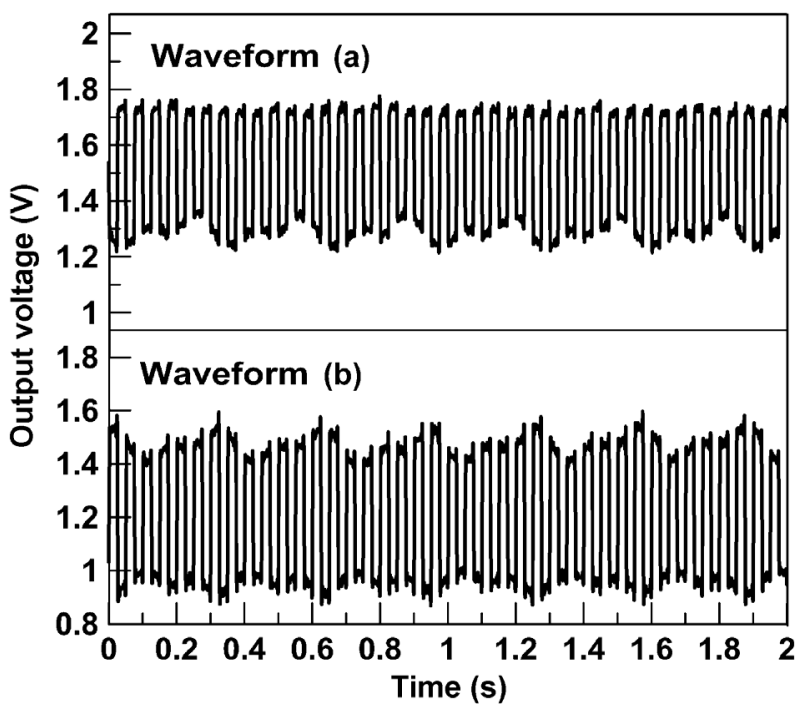

Fig. 14. Measured output voltage waveforms in the proposed path-diversity scheme.

\section{Operation of Path-Diversity Switch Transmitter}

The typical output voltage waveforms when switching periodically between the two orthogonal SIL paths are shown in Fig. 14. The copper plate is placed in front of the antennas at different distances and vibrates at a frequency of $3.2 \mathrm{~Hz}$. The period of a sensing period $T_{S F}$ is $50 \mathrm{~ms}$, which is short enough to detect vital-sign signals with periods typically larger than 500 ms. To demonstrate the orthogonality of the two SIL paths, the duty cycle of each pulse is set to $50 \%$ and the guard time is set to zero. Fig. 14(a) displays the measured output voltage waveform with one SIL path ended at the optimal point and the other ended at the null point, which is similar to the measured results at $68 \mathrm{~cm}$ distance in Fig. 9. Fig. 14(b) shows the measured output voltage waveform with both SIL paths ended neither at the optimal points nor at the null points, which is similar to the measured results at $67 \mathrm{~cm}$ distance in Fig. 9. The path-diversity transmission results in AM-like VCO tuning voltage waveforms with a carrier frequency of $20 \mathrm{~Hz}$. It can also be observed that the dc levels of the two orthogonal SIL paths are different due to the clutter.

\section{E. Vital-Sign Detection Results}

In the vital-sign detection experiments, the human subject sits in front of the prototype Doppler radar at the distances of 2 and 4 meters. The antenna beam points to the front side of the chest wall. The period of a sensing period $T_{S F}$ is $50 \mathrm{~ms}$. The two sensing pulses $T_{P 1}$ and $T_{P 2}$ have the same duration of $1 \mathrm{~ms}$, which corresponds to $2 \%$ duty cycle. The measured transition settling time between the steady-states is below $0.2 \mathrm{~ms}$ to ensure steady SIL operations. The guard time interval $T_{G}$ is set to $9 \mathrm{~ms}$ between the two sensing pulses. The sampling of the output voltage is synchronized with the control pulses. There are clutters from several stationary objects within the detection range such as tables, chairs and concrete walls in the experimental environment. The dc offsets caused by the environmental clutters and circuit coupling are removed before the detection output is 
TABLE I

Summarized Results of RecentLy Reported CW Radars For Vital Sign DeteCtion

\begin{tabular}{|c|c|c|c|c|c|c|}
\hline Authors & Radar architecture & $\begin{array}{l}\text { Solution to overcome the } \\
\text { null detection points }\end{array}$ & $\begin{array}{c}\text { Operating } \\
\text { frequency } \\
(\mathrm{GHz})\end{array}$ & $\begin{array}{c}\text { Transmitted } \\
\text { power } \\
(\mathrm{dBm})\end{array}$ & $\begin{array}{c}\text { Antenna } \\
\text { gain } \\
(\mathrm{dBi})\end{array}$ & $\begin{array}{c}\text { Maximum } \\
\text { detection } \\
\text { distance }(\mathrm{m})\end{array}$ \\
\hline Droitcour et al. [4] & Direct-conversion receiver & Quadrature receiver & 2.4 & 10 & 8 & 0.5 \\
\hline Li et al. [6] & Direct-conversion receiver with $2 \times 2$ antenna array & Quadrature receiver & 5.8 & 7 & N/A & 3 \\
\hline Xiao et al. [9] & Double-sideband transmission & $\begin{array}{c}\text { Heterodyne receiver with IF } \\
\text { frequency tuning }\end{array}$ & 27 & -19 & 12.9 & 2 \\
\hline Wang et al. [10] & Self-injection locked oscillator & Frequency sweeping & 3.6 & 0 & 12 & 4 \\
\hline Bakhtiari et al. [14] & Millimeter wave sensor & Quadrature receiver & 97 & 15 & 43 & 5 \\
\hline Chuang et al. [15] & Millimeter wave sensor with clutter cancellation & N/A & 60 & 8 & 25 & 2 \\
\hline Singh et al. [16] & Harmonic Doppler radar with passive RF tags & Quadrature receiver & 2.45 & 10 & 5 & 1 \\
\hline Kim et al. [27] & Oscillation frequency deviation & N/A & 2.4 & 4.7 & N/A & 0.02 \\
\hline Myoung et al. [28] & Direct conversion receiver with phase-locked oscillator & Quadrature receiver & 2.4 & 0 & 0 & 0.5 \\
\hline This work & Phase- and self-injection-locked oscillator & Path-diversity switch & 2.4 & -22 & 12 & 4 \\
\hline
\end{tabular}

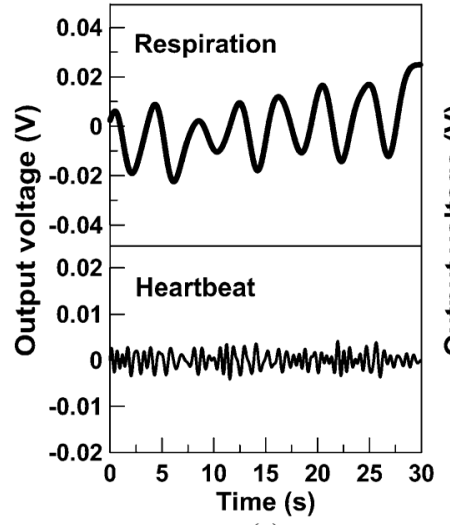

(a)

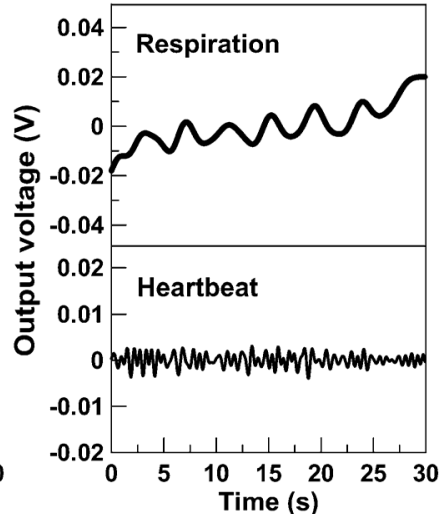

(b)
Fig. 15. Measured vital-sign voltage waveforms of the PSIL radar with the path-diversity switch transmitter at the range of (a) $2 \mathrm{~m}$ (b) $4 \mathrm{~m}$.

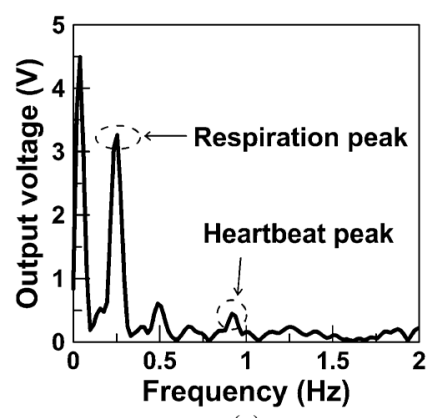

(a)

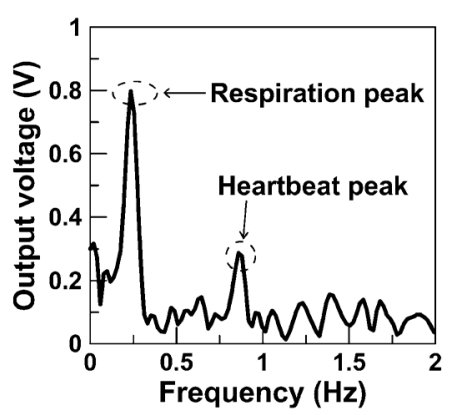

(b)
Fig. 16. Measured frequency spectra of the PSIL radar with the path-diversity switch transmitter at the range of (a) $2 \mathrm{~m}$ (b) $4 \mathrm{~m}$.

filtered to extract the respiration and the heartbeat signals. The complex signal demodulation approach [13] can be applied for obtaining more information of the signal components from the two orthogonal SIL paths.
Fig. 15 shows the filtered respiration and heartbeat waveforms of the human subject at the distance of 2 and $4 \mathrm{~m}$, respectively. The obvious variation in spectral peaks observed at very low frequencies is primarily due to the random body movement. The respiration and the heartbeat rates are determined by the Fourier analysis of the time domain waveforms. Fig. 16 shows the frequency spectra at the distance of 2 and $4 \mathrm{~m}$, respectively. The respiration and the heartbeat rate are 13 beats/min and 55 beats/min, respectively. Table I lists the results of recently reported Doppler radars for vital-sign detection. The present proposed PSIL radar has the maximum detection distance to transmitted power ratio among all recently reported works.

\section{CONCLUSION}

A PSIL radar with simple and robust de offset elimination technique has been presented for cardiopulmonary activity detection. The system complexity has been reduced by avoiding the demodulators and complicated RF and DSP clutter cancellation circuits. The circuit parameters can be quickly obtained from the design equations for specified detection sensitivity. Better phase noise performance is achieved by both the phase locked architecture and self-injection mechanism. Path-diversity transmission provides two orthogonal SIL paths to eliminate the null points and meanwhile reduce the average transmitted power.

The prototype circuit implemented by the hybrid dual-tuning VCO and the commercial PLL synthesizer IC has proven the feasibility of the proposed techniques. At $2.4 \mathrm{GHz}$ operating frequency with $-22 \mathrm{dBm}$ average transmitted power, the prototype circuit can detect the respiration and the heartbeat rates within 4 meters range.

\section{REFERENCES}

[1] J. C. Lin, "Noninvasive microwave measurement of respiration," Proc. IEEE, vol. 63, p. 1530, Oct. 1975. 
[2] K.-M. Chen, D. Misra, H. Wang, H.-R. Chuang, and E. Postow, "An $\mathrm{X}$-band microwave life-detection system," IEEE Trans. Biomed. Eng., vol. 33, pp. 697-701, Jul. 1986.

[3] K.-M. Chen, Y. Huang, J. Zhang, and A. Norman, "Microwave lifedetection system for searching human subjects under earthquake rubble or behind barrier," IEEE Trans. Biomed. Eng., vol. 47, pp. 105-114, Jan. 2000.

[4] A. D. Droitcour, O. Boric-Lubecke, V. M. Lubecke, J. Lin, and G. T. A. Kovacs, "Range correlation and I/Q performance benefits in single-chip silicon Doppler radars for noncontact cardiopulmonary monitoring," IEEE Trans. Microw. Theory Tech., vol. 52, pp. 838-848, Mar. 2004.

[5] A. D. Droitcour, O. Boric-Lubecke, and G. T. A. Kovacs, "Signal-tonoise ratio in Doppler radar systems for heart and respiratory rate measurements," IEEE Trans. Microw. Theory Tech., vol. 57, pp. 2498-2507, Oct. 2009

[6] C. Li, X. Yu, C.-M. Lee, D. Li, L. Ran, and J. Lin, "High-sensitivity software-configurable $5.8-\mathrm{GHz}$ radar sensor receiver chip in 0.13 $\mu \mathrm{m}$ CMOS for noncontact vital sign detection," IEEE Trans. Microw. Theory Tech., vol. 58, pp. 1410-1419, May 2010.

[7] H.-R. Chuang, Y.-F. Chen, and K.-M. Chen, "Automatic clutter-canceller for microwave life-detection systems," IEEE Trans. Instrum. Meas., vol. 40, pp. 747-750, Aug. 1991.

[8] T. Y. Chin, K. Y. Lin, S. F. Chang, and C. C. Chang, "A fast clutter cancellation method in quadrature Doppler radar for noncontact vital signal detection," in Proc. IEEE MTT-S Int. Microw. Symp. Dig., Jun. 2010, pp. 764-767.

[9] Y. Xiao, J. Lin, O. Boric-Lubecke, and V. M. Lubecke, "Frequencytuning technique for remote detection of heartbeat and respiration using low-power double-sideband transmission in the Ka-band," IEEE Trans. Microw. Theory Tech., vol. 54, pp. 2023-2032, May 2006.

[10] F.-K. Wang, C.-J. Li, C.-H. Hsiao, T.-S. Horng, J. Lin, K.-C. Peng, J.-K. Jau, J.-Y. Li, and C.-C. Chen, "A novel vital-sign sensor based on a self-injection-locked oscillator," IEEE Trans. Microw. Theory Tech., vol. 58, pp. 4112-4120, Dec. 2010.

[11] J. H. Oum, D.-W. Kim, and S. Hong, "Two frequency radar sensor for non-contact vital signal monitor," in Proc. IEEE MTT-S Int. Microw. Symp. Dig., Jun. 2008, pp. 919-922.

[12] B.-K. Park, O. Boric-Lubecke, and V. M. Lubecke, "Arctangent demodulation with DC offset compensation in quadrature Doppler radar receiver systems," IEEE Trans. Microw. Theory Tech., vol. 55, pp. 1073-1079, May 2007.

[13] C. Li and J. Lin, "Complex signal demodulation and random body movement cancellation techniques for non-contact vital sign detection," in Proc. IEEE MTT-S Int. Microw. Symp. Dig., Jun. 2008, pp. $567-570$.

[14] S. Bakhtiari, T. W. Elmer, II, N. M. Cox, N. Gopalsami, A. C. Raptis, S. Liao, I. Mikhelson, and A. V. Sahakian, "Compact millimeter-wave sensor for remote monitoring of vital signs," IEEE Trans. Instrum. Meas., vol. 61, pp. 830-841, Mar. 2012.

[15] H.-R. Chuang, H.-C. Kuo, F.-L. Lin, T.-H. Huang, C.-S. Kuo, and Y.-W. Ou, "60-GHz millimeter-wave life detection system (MLDS) for noncontact human vital-signal monitoring," IEEE Sensors J., vol. 12, pp. 602-609, Mar. 2012

[16] A. Singh and V. M. Lubecke, "Respiratory monitoring and clutter rejection using a CW Doppler radar with passive RF tags," IEEE Sensors $J$., vol. 12, pp. 558-565, Mar. 2012.

[17] D. Zito, D. Pepe, M. Mincica, F. Zito, A. Tognetti, A. Lanatà, and D. De Rossi, "SoC CMOS UWB pulse radar sensor for contactless respiratory rate monitoring," IEEE Trans. Biomed. Circuits Syst., vol. 5, pp. 503-510, Dec. 2011

[18] D. Girbau, A. Lázaro, Á. Ramos, and R. Villarino, "Remote sensing of vital signs using a Doppler radar and diversity to overcome null detection," IEEE Sensors J., vol. 12, pp. 512-518, Mar. 2012

[19] W. Pan, J. Wang, J. Huangfu, C. Li, and L. Ran, "Null point elimination using RF phase shifter in continuous-wave Doppler radar system," Electron. Lett., vol. 47, no. 21, pp. 1196-1198, Oct. 2011.

[20] F.-K. Wang, C.-J. Li, C.-H. Hsiao, T.-S. Horng, J. Lin, K.-C. Peng, J.-K. Jau, J.-Y. Li, and C.-C. Chen, "An injection-locked detector for concurrent spectrum and vital sensing," in Proc. IEEE MTT-S Int. Microw. Symp. Dig., Jun. 2010, pp. 768-771.

[21] A. Nezirović, A. G. Yarovoy, and L. P. Ligthart, "Signal processing for improved detection of trapped victims using UWB radar," IEEE Trans. Geosci. Remote Sens., vol. 48, pp. 2005-2014, Apr. 2010.
[22] A. N. Gaikwad, D. Singh, and M. J. Nigam, "Application of clutter reduction techniques for detection of metallic and low dielectric target behind the brick wall by stepped frequency continuous wave radar in ultra-wideband range," IET Radar Sonar Navig., vol. 5, pp. 416-425, Apr. 2011.

[23] P.-H. Wu, J.-K. Jau, C.-J. Li, T.-S. Horng, and P. Hsu, "Vital sign detection Doppler radar based on phase locked self-injection oscillator," in Proc. IEEE MTT-S Int. Microw. Symp. Dig., Jun. 2012.

[24] R. Alder, "A study of locking phenomena in oscillators," Proc. IRE., vol. 34, pp. 351-357, Jun. 1946.

[25] B. Razavi, "A study of injection locking and pulling in oscillators," IEEE J. Solid-State Circuits, vol. 39, pp. 1415-1424, Sep. 2004.

[26] C.-J. Li, C.-H. Hsiao, F.-K. Wang, T.-S. Horng, and K.-C. Peng, "A rigorous analysis of a phase-locked oscillator under injection," IEEE Trans. Microw. Theory Tech., vol. 58, pp. 1391-1400, May 2010.

[27] S.-G. Kim, G.-H. Yun, and J.-G. Yook, "Compact vital signal sensor using oscillation frequency deviation," IEEE Trans. Microw. Theory Tech., vol. 60, pp. 393-400, Feb. 2012.

[28] S.-S. Myoung, J.-H. Park, J.-G. Yook, and B.-J. Jang, "2.4 GHz Bioradar system with improved performance by using phase-locked loop," Microw. Opt. Techn. Let., vol. 52, no. 9, pp. 2074-2076, Sep. 2010.

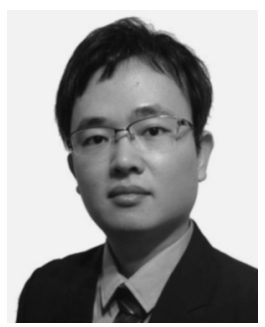

Ping-Hsun Wu (S'10) was born in Kaohsiung, Taiwan. He received the B.S. degree in electrical engineering in 2003, and the M.S. degree in communication engineering in 2005, both from the National Taiwan University, Taipei, Taiwan, where he is currently working toward the Ph.D. degree in communication engineering.

Since 2006, he has been an RF Circuit Design Engineer with the Information and Communications Research Laboratories, Industrial Technology Research Institute (ITRI), Hsinchu, Taiwan. His current research interests include the design and analysis of microwave antennas, microwave and millimeter-wave integrated circuits, and their applications in wireless and bio-electronic systems.

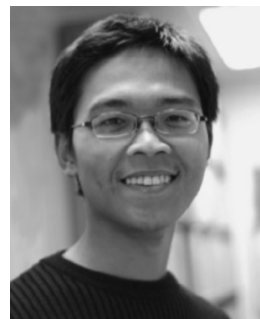

Je-Kuan Jau was born in Tainan, Taiwan, on November 19, 1977. He received the B.S.E.E. and $\mathrm{Ph} . \mathrm{D}$. degrees from National Sun Yat-Sen University, Kaohsiung, Taiwan, in 1999 and 2006, respectively.

$\mathrm{He}$ is currently an Engineer with the Information and Communications Research Laboratories, Industrial Technology Research Institute, Hsinchu, Taiwan. His research interests are RF transmitters and power amplifiers.

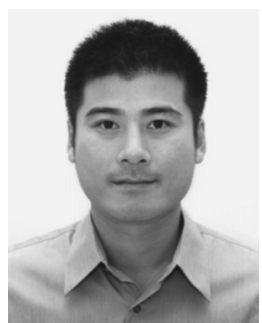

Chien-Jung Li (S'07-M'10) was born in Tainan, Taiwan, on October 26, 1979. He received the B.S.E.E. and Ph.D. degrees from National Sun Yat-Sen University, Kaohsiung, Taiwan, in 2002 and 2009, respectively.

He was a Postdoctoral Fellow with the Department of Electrical Engineering, National Sun Yat-Sen University, in 2009. After the postdoctoral position, he joined MediaTek Inc., Hsinchu, Taiwan, as a Senior Engineer, in 2010. He is currently an Assistant Professor with the Department of Electronic Engineering, National Taipei University of Technology, Taipei, Taiwan. His research interests include power amplifier (PA) linearization techniques, frequency synthesizer designs, RF sensing circuits, injection-locking techniques, and $\mathrm{LO}$ pulling issues in direct-conversion transceivers. 


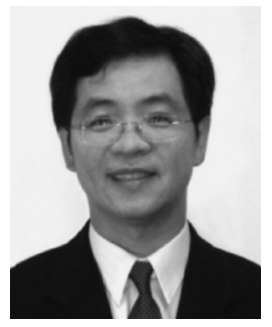

Tzyy-Sheng Horng (S'88-M'92-SM'05) was born in Taichung, Taiwan, on December 7, 1963. He received the B.S.E.E. degree from National Taiwan University, Taipei, Taiwan, in 1985, and the M.S.E.E. and Ph.D. degrees from the University of California at Los Angeles (UCLA), in 1990 and 1992, respectively.

Since August 1992, he has been with the Department of Electrical Engineering, National Sun Yat-Sen University, Kaohsiung, Taiwan, where he was the Director of the Telecommunication Research and Development Center (2003-2008) and Director of the Institute of Communications Engineering (2004-2007), and where he is currently a Distinguished Professor. He has authored or coauthored over 200 technical publications published in refereed journals and conferences proceedings, mostly in IEEE publications. He holds over ten patents. His research interests include RF and microwave ICs and components, RF signal integrity for wireless system-in-package, digitally assisted RF technologies, and green radios for cognitive sensors and Doppler radars.

Dr. Horng has served on several Technical Program Committees of international conferences including the International Association of Science and Technology for Development (IASTED) International Conference on Wireless and Optical Communications, the IEEE Region 10 International Technical Conference, the IEEE International Workshop on Electrical Design of Advanced Packaging and Systems (EDAPS), the Asia-Pacific Microwave Conference (APMC), the IEEE Radio and Wireless Symposium (RWS), and the Electronic Components and Technology Conference (ECTC). He has also served on the Project Review Board in the Programs of Communications Engineering and Microelectronics Engineering at the National Science Council, Taiwan. He was the recipient of the 1996 Young Scientist Award presented by the International Union of Radio Science, the 1998 Industry-Education Cooperation Award presented by the Ministry of Education, Taiwan, and the 2010 Distinguished Electrical
Engineer Award presented by the Chinese Institute of Electrical Engineering, Kaohsiung Branch, Taiwan. Recently, he was awarded with the 2011 Advanced Semiconductor Engineering (ASE) Inc. Chair Professorship and the 2012 Outstanding Research Award at the National Sun Yat-Sen University. Dr. Horng is the Founder Chair of the IEEE MTT-S Tainan Chapter, and currently an Associate Editor of the IEEE TRANSACTIONS ON MicROWAVE THEORY AND TECHNIQUES, and a member of the IEEE MTT-S Technical Committee MTT-10 and MTT-20.

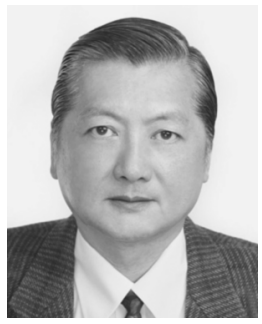

Powen Hsu (M'86-SM'98-F'10) was born in Taipei, Taiwan. He received the B.S. degree in physics from the National Tsing-Hua University, Hsinchu, Taiwan, in 1972, the M.S. degree in physics from the University of Maryland, College Park, in 1976, and the M.S. and Ph.D. degrees in electrical engineering from the University of Southern California, Los Angeles, in 1978 and 1982, respectively.

From 1982 to 1984 , he was with ITT Gilfillan, Van Nuys, CA, where he was engaged in research and development pertaining to radar antenna systems. In 1984, he joined the faculty of the National Taiwan University, Taipei, Taiwan, where he is currently a Professor with the Electrical Engineering Department. From 1992 to 1995, he was the Department Chairperson there. In August 1997, he established the ninth college, College of Electrical Engineering and Computer Science, in the National Taiwan University, and served as the first Dean of the College until 2003. His current research interests include the design and analysis of slot antennas, microstrip antennas, and microwave and millimeterwave integrated circuits.

Dr. Hsu is a Distinguished Professor of National Taiwan University. 